Research Paper

\title{
High Expression of Plakoglobin Promotes Metastasis in Invasive Micropapillary Carcinoma of the Breast via Tumor Cluster Formation
}

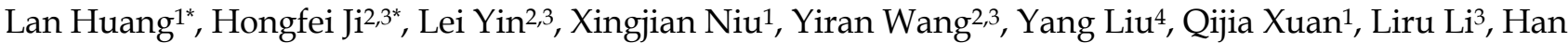 \\ Zhang1, Xiaoping Zhou ${ }^{1}$, Jingtong Li ${ }^{1}$, Chengwei Cui ${ }^{1}$, Yue Yang ${ }^{2,3}$, Weiwei Ann, ${ }^{2,3}$ Qingyuan Zhang1,2,3凶 \\ 1. Department of Medical Oncology, Harbin Medical University Cancer Hospital, Harbin Medical University, Harbin 150081, Heilongjiang, China \\ 2. Institute of Cancer Prevention and Treatment, Harbin Medical University, Harbin 150081, Heilongjiang, China \\ 3. Heilongjiang Academy of Medical Sciences, Harbin 150081, Heilongjiang, China \\ 4. Department of Breast Surgery, Harbin Medical University Cancer Hospital, Harbin 150081, Heilongjiang, China \\ *Equal contributions \\ $\triangle$ Corresponding author: jihongfei@hrbmu.edu.cn (Email); +86-451-86298333 (Phone); +86-451-86298333 (Fax) \\ (C) Ivyspring International Publisher. This is an open access article distributed under the terms of the Creative Commons Attribution (CC BY-NC) license \\ (https:// creativecommons.org/licenses/by-nc/4.0/). See http://ivyspring.com/terms for full terms and conditions.
}

Received: 2018.11.11; Accepted: 2019.04.06; Published: 2019.06.02

\begin{abstract}
Invasive micropapillary carcinoma of the breast (IMPC) is a rare subtype of breast cancer that has a high frequency of lymph node (LN) involvement and metastasis to distant organs. IMPC is characterized by distinct histomorphology and unfavorable prognosis when compared with invasive ductal carcinoma no special type (IDC-NST). However, the underlying molecular mechanisms remain unclear. We reported here that plakoglobin, as a key component in cell adhesion, can promote collective metastasis through facilitating IMPC clusters formation. In comparing the clinicopathological features of 451 IMPC patients and 282 IDC-NST patients, our results showed that tumor emboli were significantly higher in IMPC patients and were associated with a high frequency of metastasis. Both in vitro and in vivo data showed overexpression of plakoglobin in both the cell membrane and the cytoplasm of IMPC clusters. When plakoglobin was knocked down in IMPC cell models, the tumor cell clusters were depolymerized. Using mouse models, we validated the metastatic potential of tumor clusters was higher than single cells in vivo. Further analysis showed that higher expression of plakoglobin was able to promote activation of the PI3K/Akt/Bcl-2 pathway, which might protect the clusters from anoikis. Our data indicate that plakoglobin promotes tumor cluster formation in IMPC and downregulates apoptosis in the cell clusters through activation of $\mathrm{PI} 3 \mathrm{~K} / \mathrm{Akt} / \mathrm{Bcl}-2$ signaling. These results provide a convincing rationale for the high metastatic propensity seen in IMPC.
\end{abstract}

Key words: Invasive micropapillary carcinoma of the breast (IMPC), Plakoglobin, Metastasis, Anti-anoikis, PI3K pathway

\section{Introduction}

Breast cancer is the most common malignancy globally for women. It is a heterogeneous disease with substantial diversity in histological and molecular characteristics. Invasive micropapillary carcinoma of the breast (IMPC) accounts for $5 \%$ of all breast cancers, and displays relatively uncommon and distinct histomorphological features [1]. This subtype often has a poor prognosis with the $5 \mathrm{y}$ disease survival rate being $40 \%$ versus $88 \%$ for invasive ductal carcinoma no special type (IDC-NST). This is due to the high propensity of lymph node (LN) involvement as well as early regional and distant metastasis seen in IMPC [1-5]. Many aspects of IMPC have previously been studied, including clinicopathological features, analysis of prognostic factors, and relative special markers [6-16]. 
Nevertheless, a better understanding of the molecular events underlying the metastasis of IMPC will be important for its prevention, diagnosis, and treatment.

Metastatic spread is responsible for the majority of cancer-associated deaths and is affected by a variety of factors, including the deregulation of gene expression, signal transduction, and epigenetic modification, as well as epithelial-to-mesenchymal transition (EMT) and the transformation of tumor cells into stem/progenitor cells. Invading cancer cells can utilize different modes of migration including single cell migration and collective migration. Collective migration differs from single cell migration in that cells remain connected as they move. In addition, studies have demonstrated that the tumor cell clusters in circulation are neither result from the proliferation of a single tumor cell nor the aggregation of intravascular tumor cells, but are directly shed from the primary disease. Considering that previous studies have reported that IMPC displays a distinct histomorphology with small clusters and has a high frequency of tumor emboli within vessels, we speculated that the adherens junctions may be the key regulator of clusters maintain. Although in recent years, the importance of adherens junctions in metastasis has attracted much attention, the pathological relevance and significance of plakoglobin overexpression in IMPC remains elusive. Plakoglobin is a member of the Armadillo family of proteins and is a critical constituent of both adherens junctions and desmosomes. Both adherens junctions and desmosomes are cadherin-based cell adhesion complexes. In adherens junctions, plakolgobin or $\beta$-catenin interacts with E-cadherin intercellular domain in a mutually exclusive manner. Additionally, these catenin proteins play important roles in regulating tumor morphogenesis and metastasis progression by interacting with elements of cell signaling pathways. It has been demonstrated that plakoglobin can be divided into soluble and insoluble pools according to the difference in the phosphorylation levels in its amino acids, suggesting that a cellular function independent of cell adhesion it may have. High plakoglobin expression has been reported to associate with adverse clinical outcomes in breast cancer patients [17-20]. Evidence has shown that the gene product of plakoglobin can be a major determinant of tumor dissemination. In contrast, knockdown of plakoglobin has been shown to suppress cell aggregation and tumor metastasis formation [21]. Thus, we speculated that plakoglobin expression might change in IMPC clusters and contribute to the unique mechanism of collective metastasis in IMPC.
This study is the first to investigate the role of plakoglobin in IMPC cluster formation and distant metastasis. We found that plakoglobin was upregulated in both IMPC clinical samples and cell models. High plakoglobin expression was associated with decreased overall survival (OS). Knockdown of plakoglobin inhibited the cellular metastatic potential of our IMPC cell line model. Moreover, plakoglobin further enhanced IMPC cluster survival in vitro and in vivo by targeting the $\mathrm{PI} 3 \mathrm{~K} / \mathrm{Akt} / \mathrm{Bcl}-2$ pathway. Inhibiting the expression of plakoglobin significantly downregulated the expression of PI3K, Akt, and Bcl-2. Restoration of plakoglobin expression retained the high metastasis potential of IMPC clusters in mouse models. Collectively, we identified that plakoglobin functions as an oncoprotein, not only strengthening tumor cell aggregation and cluster formation, but also enhancing anoikis-suppression by activating the PI3K/Akt/Bcl-2 pathway. These findings show that plakoglobin functions as a tumor cluster regulator and metastasis promoter in IMPC development, which could provide a promising prognostic and therapeutic strategy for IMPC treatment.

\section{Materials \& Methods}

\section{Patients and cohort}

Breast cancer patients (451 IMPC patients and 282 IDC-NST patients) diagnosed between January 2009 to December 2013 in Harbin Medical University Cancer Hospital (Harbin, China) were identified by retrieving their electronic medical records. Their pathological diagnosis, LN involvement, local recurrence, distant metastasis, and follow-up information were reviewed. IMPC diagnosis was based on the morphological criteria described in the World Health Organization (WHO) histological classification. Follow-up information, including tumor relapse and survival status, was available through treatment records or personal contact with the patients via telephone calls. This study was approved by the Scientific and Ethical Committee of the Cancer Hospital/Institute of Harbin Medical University, and all patients provided written informed consent.

\section{Cell culture}

Human breast cancer cells, T47D and MCF7, were obtained from the American Type Culture Collection (ATCC). The cell lines were tested and authenticated by the standard short tandem repeat DNA typing methodology before use in this study. T47D and MCF7 were cultured in RPMI-1640 (GIBCO) medium containing $10 \%$ fetal bovine serum (FBS, GIBCO), $100 \mathrm{U} / \mathrm{mL}$ penicillin, and $50 \mu \mathrm{g} / \mathrm{mL}$ streptomycin, and grown in a $5 \% \mathrm{CO}_{2}$ humidified 
incubator at $37^{\circ} \mathrm{C}$. The cells were last tested on July 2016.

\section{Cellular spheroid formation via neutrophil elastase}

Cells were proliferated into an exponential phase and treated with $0.25 \%$ trypsin (1:250; GIBCO) to detach them from the culture medium. Then FBS was added to inactivate the trypsin and the cells were washed once with medium. After cell counting, $5 \times 10^{5}$ cells were added to each well of a 24-well plate (Corning) and cultured with $20 \mu \mathrm{g} / \mathrm{mL}$ human neutrophil elastase (Calbiochem) under the above conditions. After $24-48$ h of incubation, cell clusters were observed [22-23].

\section{Immunohistochemistry analysis and scoring for plakoglobin}

Immunohistochemistry (IHC) was performed using the streptavidin-perosidase method with mouse monoclonal antibodies against CD31 (Abcam), D2-40 (Abcam) and Bcl-2 (Abcam); rabbit monoclonal antibodies against plakoglobin (CST), PI3K p85 (Abcam) and pAKT (Ser473; CST). Sections of the tumor tissue, of $4 \mu \mathrm{m}$ in thickness, were dewaxed in xylene and rehydrated through graded alcohols. Antigen retrieval was carried out using citrate buffer (pH 6.1) at $120^{\circ} \mathrm{C}$ and $111.46 \mathrm{kPa}$ for $90 \mathrm{~s}$. To block the activity of endogenous peroxidase, $3 \%$ hydrogen peroxide was added for $20 \mathrm{~min}$ at $37^{\circ} \mathrm{C}$. Next, the primary antibodies were added and the sections were incubated at $4{ }^{\circ} \mathrm{C}$ overnight. This was followed by washing with PBS and secondary antibody incubation at $37^{\circ} \mathrm{C}$ for $30 \mathrm{~min}$. Subsequently, the sections were dyed with $3^{\prime}$-diaminobenzidine (DAB). The slides were then counterstained with hematoxylin and covered with coverslips.

Protein expression of plakoglobin, PI3K, pAkt and BCL-2 was assessed by IHC and scored semiquantitatively. Tumor sections were scored based on the following standards: the proportion score was classified as $0(<10 \%), 1(10-30 \%), 2(30-50 \%)$ or 3 $(>50 \%)$, and the staining intensity was classified as 0 (absent), 1 (weak), 2 (moderate), or 3 (dense). A total score is equal to the percentage score multiplied by intensity score. Staining was defined as 'positive' if the total score was greater than 2; otherwise, the staining was regarded as negative.

\section{Immunofluorescence staining analysis}

T47D cells $\left(5 \times 10^{5}\right)$ and T47D cell clusters grown in 24-well plates were fixed with $4 \%$ paraformaldehyde for $30 \mathrm{~min}$, then washed with PBS three times and stored at $4{ }^{\circ} \mathrm{C}$. After $24 \mathrm{~h}$, cells were permeabilized with $0.5 \%$ TritonX-100 for $5 \mathrm{~min}$, followed by washing with PBS. Cells were then incubated with an anti-human plakoglobin antibody (CST) at $4{ }^{\circ} \mathrm{C}$ overnight. The next day, cells were incubated with the secondary antibody (goat anti-rabbit IgG $(\mathrm{H}+\mathrm{L})$ conjugated to cy3 Fluor dye (Invitrogen)) for $30 \mathrm{~min}$, and treated with PBS and a gradient of ethanol for $5 \mathrm{~min}$ each. The cells were incubated with DAPI for $5 \mathrm{~min}$ and covered with coverslips. Finally, they were visualized and recorded using a Leica DMi8 confocal microscope. After secondary antibody addition, all steps were performed in dark conditions.

\section{Plakoglobin knockdown by shRNA transfection}

To assess the effects of plakoglobin inhibition on cluster maintenance 2 shRNAs for plakoglobin (shRNA1 sequence 5'CCAUCGGCUUGAUCAGGA Att-3', and shRNA2 sequence 5'GGGCAUCAUGGAG GAGGAUtt-3) and a negative control shRNA (CTRL shRNA sequence) (GeneChem, Shanghai, China) were utilized. T47D and MCF7 cell clusters were formed and cultured in a 24-well plate as described above. Cell clusters were infected with lentiviruses expressing either CTRL shRNA or shRNA1/shRNA2 for knocking down plakoglobin. Transduced tumor cell clusters were sorted by $1 \mathrm{ug} / \mathrm{mL}$ puromycin. shRNA2 was more efficient at knocking down plakoglobin and was therefore used in the following experiments.

\section{Western blot analysis}

Equal amount of cell extracts were obtained from T47D/MCF7 single cells and T47D/MCF7 cell clusters. These were separated by $8 \%$ SDS-PAGE and transferred to nitrocellulose membranes. After blocking with $5 \%$ nonfat dry milk/Tris-buffered saline solution (TBS), the blots were incubated with anti-human plakoglobin (CST), PI3K p85 (Abcam), pAkt (phospho Ser473; CST), Bcl-2 (Abcam), Caspase-3 (Abcam), or $\beta$-actin (Abcam) antibodies at 4 ${ }^{\circ} \mathrm{C}$ overnight. The antigens were visualized by peroxidase-conjugated secondary antibodies and densitometry was performed by Advanced Image Data Analyzer software v4.5 (Raytek Scientific).

\section{In vivo metastasis}

A total of 20 female immunodeficient (NSG) mice (8-weeks old) were included in the test and were randomized into two groups ( $\mathrm{n}=10$; Vital River). Equal cell numbers $\left(2 \times 10^{6}\right)$ of T47D single cells and T47D cell clusters were injected via the tail vein into mice respectively. After 3 weeks, the mice were sacrificed and the lungs were excised. The lungs were embedded in paraffin, subjected to serial sections and stained with hematoxylin-eosin (H\&E). Paraffin sections from the same lobe and same location were 
selected. Five random areas per section were photographed and counted the number of metastatic foci. All of the procedures involving the care and use of laboratory animals were performed in accordance with institutional and national guidelines. Animal studies were approved by Harbin Medical University Institutional Animal Care and Use Committee.

\section{Anoikis induction and flow cytometry}

In order to induce anoikis, T47D $\left(1 \times 10^{6}\right)$ cells treated with or without neutrophil elastase were cultured in plates coated with polyhydroxyethylmethacrylate (poly-HEMA, Sigma) for $24-48 \mathrm{~h}$ at 37 ${ }^{\circ} \mathrm{C}$ and $5 \% \mathrm{CO}_{2}$. After washing (PBS, $1000 \mathrm{rpm}, 5$ $\mathrm{min})$, the cells were harvested and separated by cell strainers (BD Biosciences). Next, $500 \mu \mathrm{l}$ binding buffer was added to each sample and they were incubated on ice for $30 \mathrm{~min}$. According to the manufacturer's protocol, cells were stained with propidium iodide and with the Annexin V-FITC apoptosis detection kit (Wan Lei bio Co.). Cells were sorted using BD FACS Accuri C6 flow cytometry (Becton-Dickinson), and CELLQuest (Becton-Dickinson) software was used to analyze the data.

\section{Statistical analysis}

Results were presented as mean \pm SD. Pearson's chi-squared test was used in the analysis of contingency tables; Fisher's exact test was used when needed. Comparison between two ratios was conducted by using a test of two proportions. Overall survival (OS) rates were estimated using the Kaplan-Meier method and were analyzed using the log-rank test. Multivariate analysis was performed using the Cox risk proportion model. Hazard ratios (HR) were presented with 95\% confidence intervals (CI). All statistical tests were two sided and results were considered to be statistically significant at $P$-values $<0.05\left({ }^{*} p<0.05,{ }^{* *} p<0.01,{ }^{* * *} p<0.001\right)$. The SPSS 17.0 software package and Graphpad Prism were used for statistical analysis.

\section{Results}

\section{IMPC shows a higher tumor emboli and metastasis potential compared to IDC-NST.}

In a comparison of 451 IMPC patients with 282 IDC-NST patients, we observed that the LN metastatic rate was significantly higher in IMPC than IDC-NST patients $(66.7 \%$ vs. $22.7 \%$; $P<0.001)$. Additionally, the mean number of metastatic LNs was significantly higher in IMPC patients than in IDC-NST patients, and more IMPC patients suffered distant metastasis $(P=0.038) \quad$ (Table 1$)$. When investigating the histological features of IMPC, we found that IMPC was characterized typically by small clusters or a nesting papillary pattern (Figure 1A \& B). Furthermore, tumor embolism was identified in the lymph and blood vessels by labeling with anti-D2-40 and anti-CD31 antibodies (Figure $1 C$ \& D). The positive rate of tumor emboli in the IMPC group was significantly higher than that in the IDC-NST group (25.9 \% vs. $12.4 \%$; $P<0.001)$ (Table 1$)$. These results suggest that IMPC patients have higher rates of $\mathrm{LN}$ and distant metastasis involvement than IDC-NST patients. Furthermore, these suggest that the tumor cell clusters were the main mode of metastasis, which is probably related to their high tumor emboli.

Table 1. Comparison of clinical pathology features between IMPC and IDC-NST

\begin{tabular}{llclll}
\hline & \multicolumn{2}{l}{ IMPC $(\mathbf{n = 4 5 1 )}$} & \multicolumn{3}{l}{ IDC-NST } \\
(n=282) & \\
\hline Characteristics & $\mathrm{n}$ & $\%$ & $\mathrm{n}$ & $\%$ & $P$-values \\
\hline Mean LN & 5.25 & & 3.62 & & \\
LN-pos & 301 & 66.7 & 64 & 22.7 & $<0.001$ \\
DM & 111 & 24.6 & 49 & 17.4 & 0.038 \\
TM-pos & 117 & 25.9 & 35 & 12.4 & $<0.001$ \\
\hline
\end{tabular}

LN: lymph node; LN-pos: lymph node positive; DM: distant organ metastasis; TM-pos: tumor emboli positive (intravascular tumor emboli).

\section{Plakoglobin is overexpressed in IMPC.}

Plakoglobin expression was detected in 48 IMPC patients and 50 IDC-NST patients via immunohistochemistry, and results showed higher plakoglobin expression in IMPC samples compared to IDC-NST samples (Figure 2A-D), especially in metastatic LNs (Figure 2E \& F). The clinicopathological features of 98 samples are summarized in Supplementary Table 1. Of the 48 patients with IMPC, 34 samples (70.8 \%) were observed to be positive for plakoglobin. By contrast, only 21 cases ( $42 \%$ ) of IDC-NST were positive, which is a significant statistical difference $(P=0.004$; Figure $2 \mathrm{G})$. Represented images of IHC staining of plakoglobin with various intensity displayed in Supplementary Figure 1. To estimate the clinical significance of various prognositic factors (including plakoglobin expression) that may influence all survival in IMPC, we performed multivariate Cox regression analysis. As shown in Table 2, the multivariate Cox proportional hazards regression analysis revealed that distant metastasis $(P=0.021$; $\mathrm{HR}=3.35$, 95\% CI 2.76-4.32), lymph node status $(P=0.037 ; \mathrm{HR}=2.62,95 \% \mathrm{CI} 1.78-3.86)$ and plakoglobin expression $(P=0.042 ; \mathrm{HR}=1.49,95 \%$ CI $1.07-2.43)$ were independent prognostic factors for the OS of patients with IMPC (Table 2). In addition, Kaplan-Meier survival analysis showed that plakoglobin upregulation in IMPC was closely related to poor patient survival $(P=0.002$; Figure $2 \mathrm{H})$, suggesting an important role of plakoglobin in IMPC. 

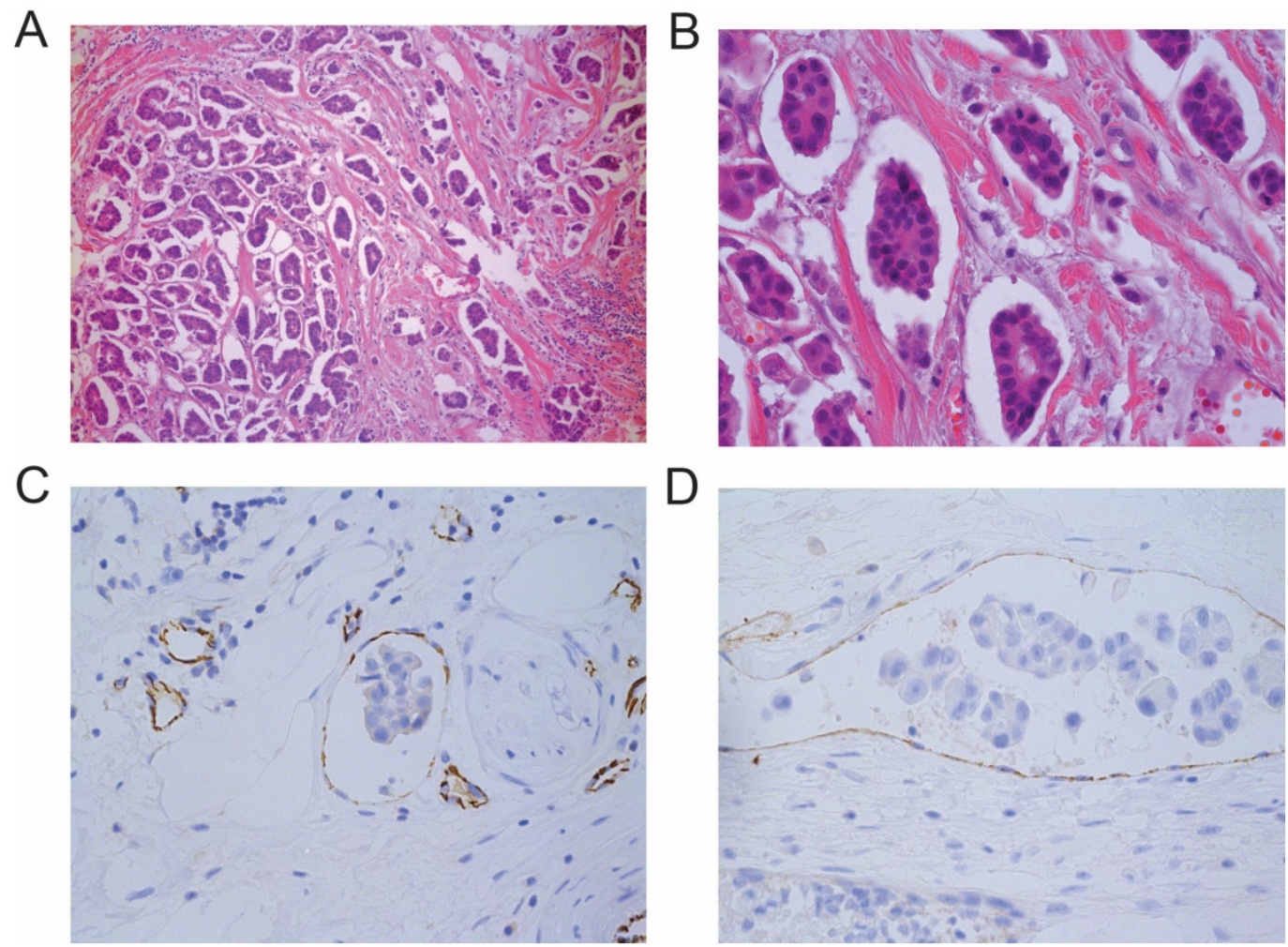

Figure 1. IMPC histomorphology feature and tumor emboli. A-B: Representative microscope images of an IMPC primary lesion with small solid-nests stained with H\&E (1A: 100x, 1B: 400x). C-D: CD31 and D2-40 staining of IMPC showing tumor embolism in lymphatic and blood vessels (400x).

Table 2. Multivariate survival analysis by Cox regression model for OS of IMPC

\begin{tabular}{llll}
\hline IMPC samples & $\boldsymbol{P}$-value & HR & $\mathbf{9 5 \%} \mathbf{C I}$ \\
\hline DM & 0.021 & 3.35 & $2.76-4.32$ \\
LN & 0.037 & 2.62 & $1.78-3.86$ \\
ER & 0.071 & 0.44 & $0.21-1.07$ \\
T stage & 0.149 & 1.07 & $0.87-1.45$ \\
Plakoglobin exp & 0.042 & 1.49 & $1.07-2.43$ \\
\hline
\end{tabular}

HR: hazard ratio; DM: Distant metastasis; LN: lymph node status; ER: estrogen receptor; T stage: tumor stage; Plakolgobin exp: plakolgobin expression.

\section{Plakoglobin is correlated with IMPC clusters and contributes to IMPC metastasis.}

To identify whether plakoglobin played a role in IMPC clusters, T47D/MCF7 cell clusters were constructed using neutrophil elastase to imitate IMPC cells in vitro, and plakoglobin expression was assessed by western blotting and immunofluorescence staining. Figure $3 \mathrm{~A} \& 3 \mathrm{~B}$ show a higher level of plakoglobin in T47D/MCF7 cell clusters compared with T47D/MCF7 single cells. In addition, plakoglobin (shown in red via the cy3 Fluor dye) was strongly expressed between cell-cell connections in T47D cell clusters. However, there was only weak expression in T47D single cells (Figure 3C). We then introduced 2 plakoglobin shRNAs (shRNA1 and shRNA2) into T47D cell clusters to knockdown plakoglobin as well as a non-target shRNA control. We observed that suppression of plakoglobin by
shRNAs triggered disruption of cell-cell contacts (Figure 3D-E). Furthermore, we performed in vivo studies by injecting T47D cell clusters or single cells respectively into immunodeficient mice via tail vein injections. As shown in Figure 4A to 4D, mice injected with T47D cell clusters displayed more lung metastasis than the controls by histopathological analysis with statistically significance $(P<0.001$, Figure $4 \mathrm{E})$. Overall, these results demonstrate that plakoglobin is probably involved in IMPC cluster formation and might contribute to the distant metastasis of IMPC.

\section{Tumor clusters formation inhibits anoikis}

Anoikis resistance is considered as a hallmark of metastatic cancer cells. We analyzed whether IMPC cluster formation enhanced their anti-anoikis ability while in circulation. To assess anoikis, the apoptotic rate of T47D cell clusters and single cells cultured in poly-HEMA coated plates was carried out by flow cytometry (Figure 5A \& B). The apoptotic rate of T47D cell clusters were significantly reduced compared to the apoptotic rate of T47D single cells. In addition, an increase in caspase-3 expression was detected in T47D single cells compared to T47D cell clusters under suspension conditions (Figure 5C). These results indicated that the formation of clusters greatly suppresses anoikis and prolongs the survival of cells under anchorage-independent conditions. 
A
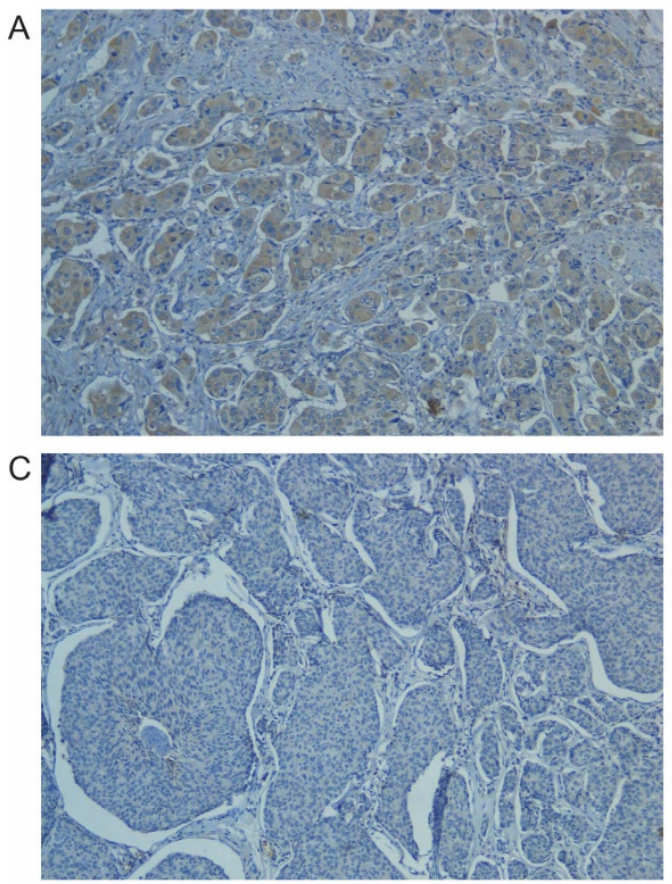

E

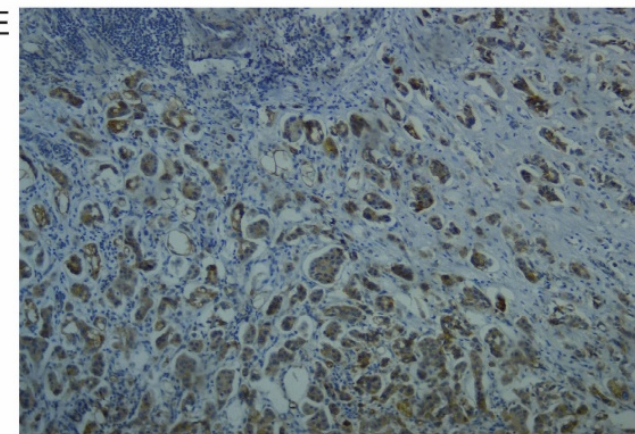

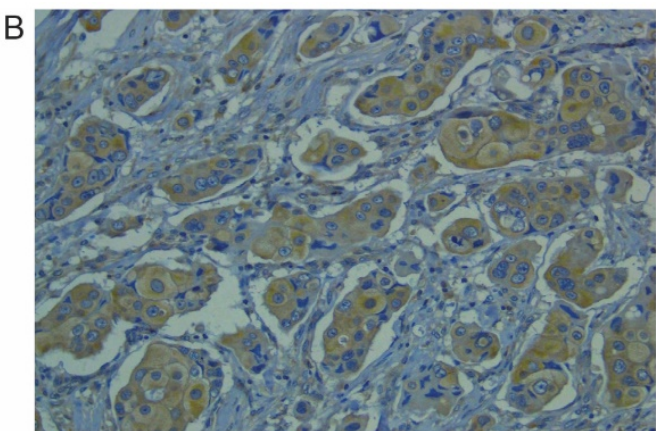
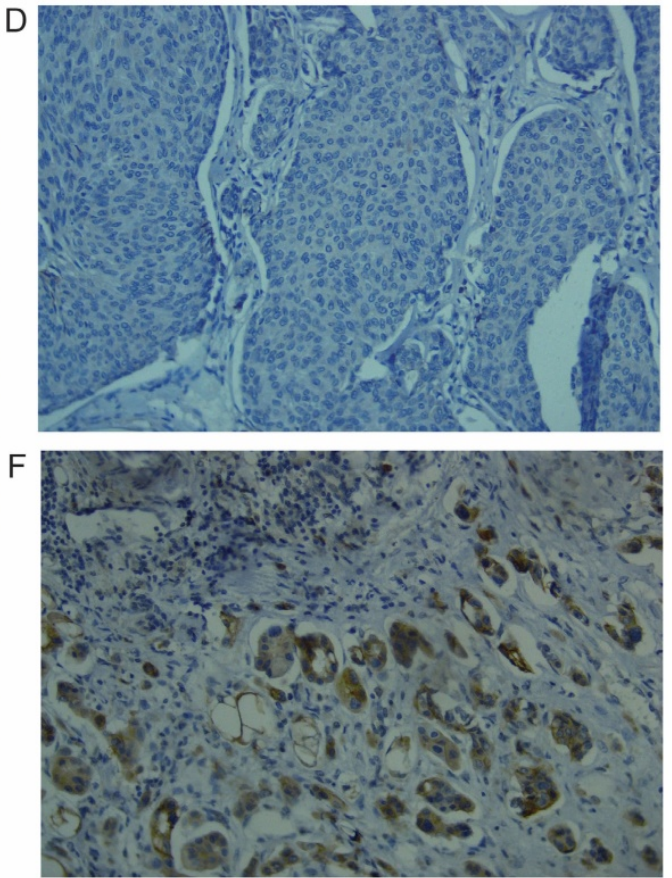

G
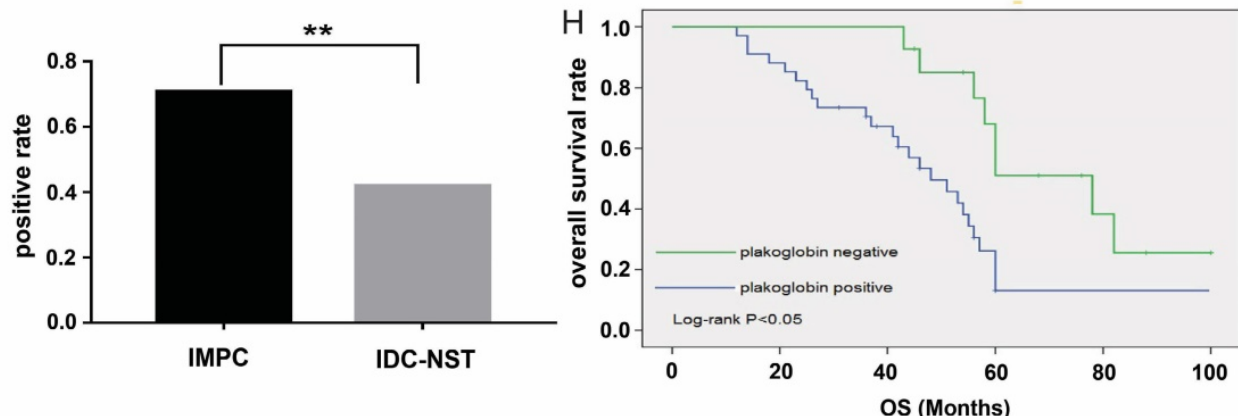

Figure 2. Plakoglobin is overexpressed in IMPC and correlates with poor prognosis. A-B: Overexpression of plakoglobin in the cytoplasm and membrane of IMPC cells (2A: 100x, 2B: 200x). C-D: Negative expression of plakoglobin in IDC cells (2C: 100x, 2D: 200x). E-F: Immunohistochemistry of plakoglobin overexpression in metastatic LNs (2E: 100x, 2F: 200x). G: Bar graph displaying the positive rate of plakoglobin staining in IMPC and IDC (**p<0.01). H: Kaplan-Meier curves for the effect of plakoglobin expression on $O S$ rate $(p=0.002)$.

\section{High plakoglobin in IMPC antagonizes anoikis via the PI3K/Akt/Bcl-2 pathway}

As mentioned above, aggregated tumor cells have a lower apoptotic rate. Additionally, we have shown that high levels of plakoglobin are detected in metastatic lesions (Figure 2E \& F), which indicates that this metastasis was directly derived from IMPC clusters. To further investigate whether this high level of plakoglobin was involved in maintaining the vitality of IMPC clusters, plakoglobin in T47D cell clusters was knocked down by shRNA and proteins relative to anoikis, including PI3K-p85, pAkt (Ser473), and $\mathrm{Bcl}-2$, which were examined by western blotting. The level of plakoglobin, PI3K-p85, pAkt and Bcl-2 were all reduced by plakoglobin shRNA transfection (Figure 3A \& B). The above results have also been varified in the MCF7 cell line and shown in Figure 3C $\&$ D. To further compare the expression profiles of PI3K-p85, pAkt (Ser473) and Bcl-2 in IMPC and 
IDC-NST, we conducted immunohistochemical staining for PI3K-p85, pAkt (Ser473) and Bcl-2 in IMPC and IDC-NST specimens. Higher expression rates of PI3K-p85, pAkt (Ser473) and Bcl-2 were observed in IMPC than IDC-NST specimens. A correlation analysis demonstrated that the expression of plakoglobin in IMPC had a positive correlation with the expression of PI3K-p85, pAkt and Bcl-2 $(P<$ 0.001) (Supplementary Table 2). These results indicate that plakoglobin overexpression might protect IMPC clusters from anoikis through activating the PI3K/Akt/Bcl-2 pathway.

\section{Discussion}

Recurrence and metastasis are the most common causes for breast cancer fatality, which generally occurs within 5 years. IMPC has been well documented as a rare breast cancer subtype with a unique histomorphology and a high risk of recurrence and metastasis. However, the underlying mechanism behind the propensity of metastasis is still largely unknown. Previous studies have reported that cell migration is a key process of tumor metastasis, and there are many different migration patterns that exist in diverse biologic contexts. Among them, cluster migration (or "collective" cell migration) is one of the

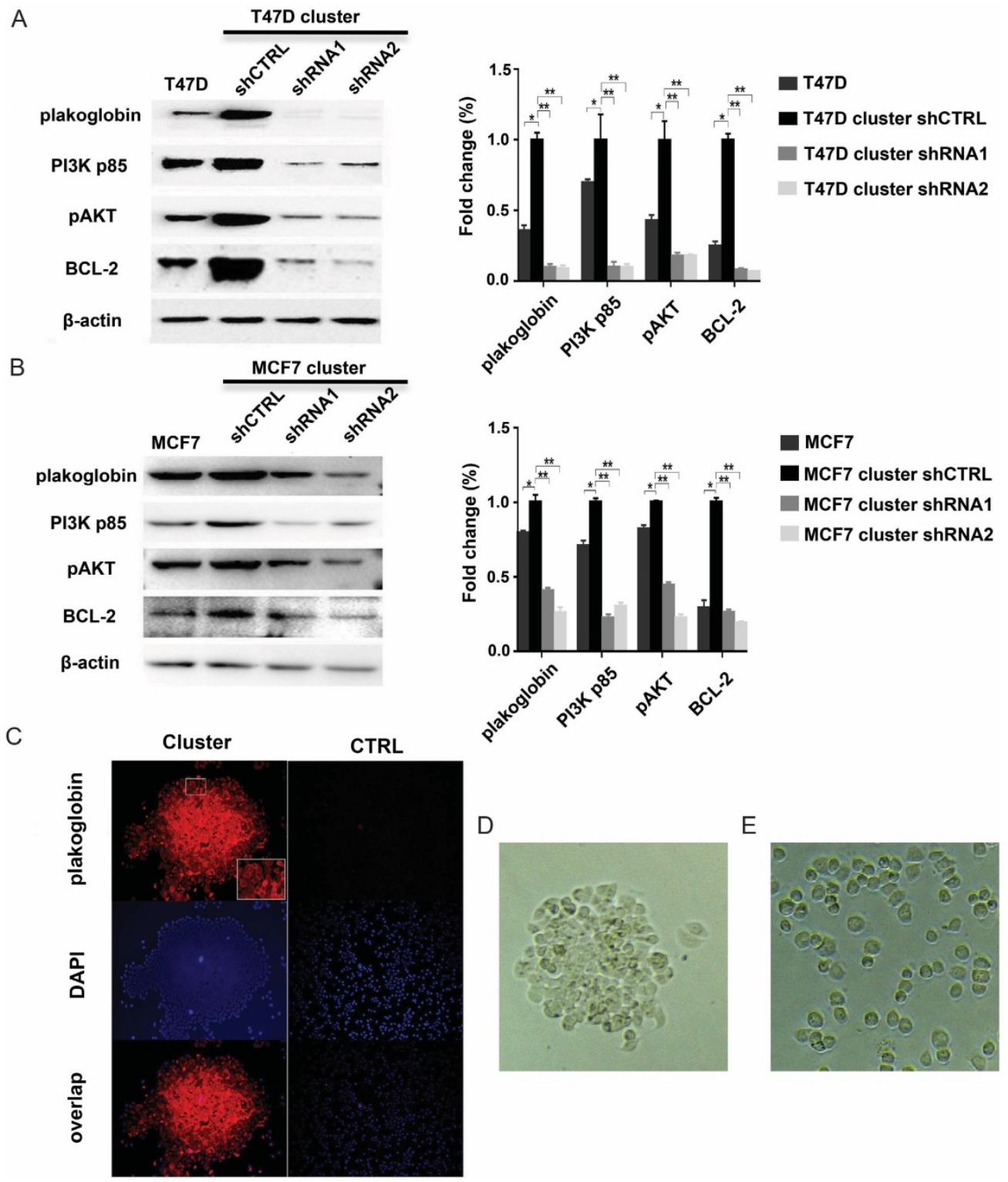

Figure 3. Plakoglobin is required for IMPC cluster formation and activates the PI3K/Akt/BCL2 pathway. A: Representative immunoblot showing the expression levels of plakoglobin, PI3K p85, pAkt, and Bcl-2 in T47D single cells as well as T47D cell clusters expressing control (shCTRL) or plakoglobin shRNAs (shRNA1 and shRNA2). $\beta$-Actin was used as a loading control (left). Error bars represent SEM. $n=3 ;{ }^{*}<<0.05 * * p<0.01$ by student's t test (right). B: The immunoblot results were verified in the MCF7 cell line (left). Error bars represent SEM. $n=3$ (right). C: Representative confocal immunofluorescence images of T47D cell clusters and T47D single cells (CTRL) stained for plakoglobin (red) and DAPI (nuclei, blue). D-E: Representative image of T47D cell cluster in vitro (3D). Its corresponding depolymerized form caused by plakoglobin shRNA transfection (3E). 
A

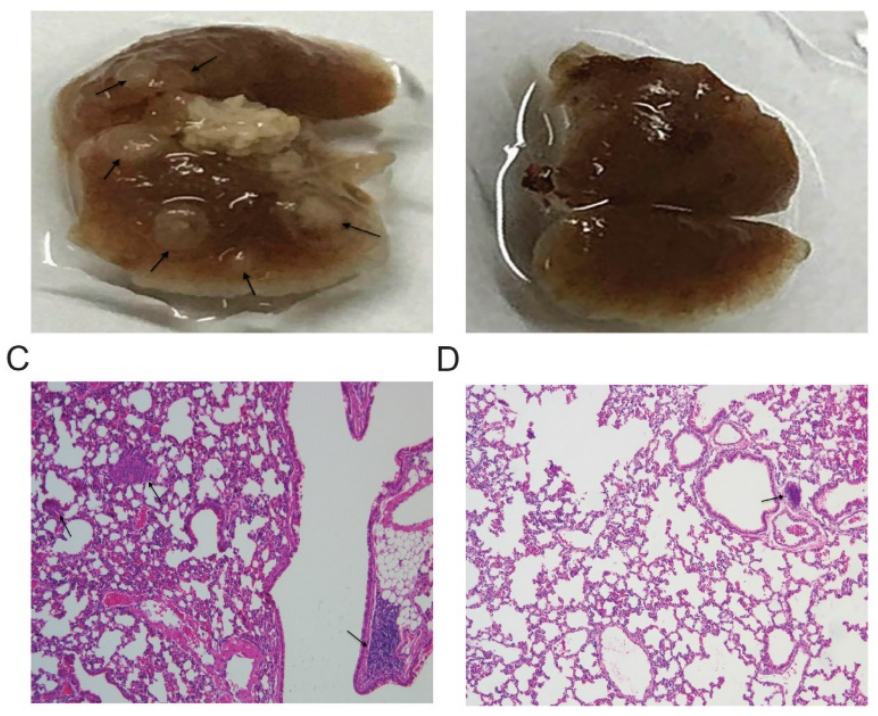

$\mathrm{E}$

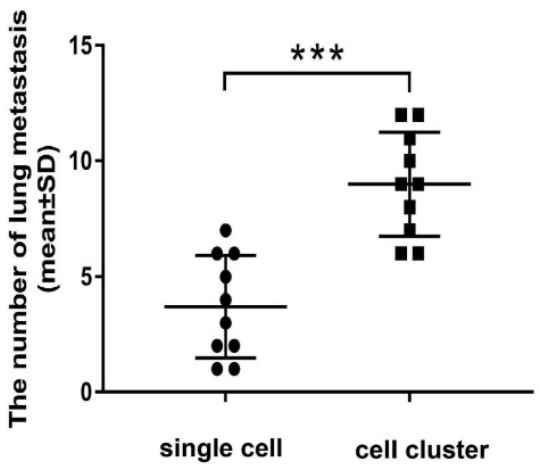

Figure 4. Lung metastatic lesions in mice injected with T47D cell clusters or T47D single cells. A-B: Representative images of lungs from mice injected with T47D cell clusters (4A) or T47D single cells (4B) via tail vein injections. Arrow indicates tumor nodule. C-D: Representative images of the lung tissues from mice injected T47D cell clusters (4C: 100x) or T47D single cells (4D: 100x) stained with H\&E. Arrow indicates tumor nodule. E: The number of lung metastases was recorded to evaluate the metastasis. Each dot represents the mean number of lung metastasis assessed by histopathological analysis per field of view. Horizontal bars indicate the median value in each group. The Student's t test was used for statistical analysis, ${ }^{* * *} p<0.001(n=10$ mice per group).

main strategies in epithelial tumor metastasis [24]. In our comparison of the clinicopathological features between 451 IMPC and 282 IDC-NST patients, we have demonstrated that the incidence of tumor emboli and metastasis was statistically higher in IMPC patients. Previous studies have observed that the epithelial-mesenchymal transition (EMT) was occurred in IMPC cell clusters and may contribute to the invasion and metastasis of IMPC in clustered form [25-26]. Given that the histopathological morphology of IMPC is very similar to intravascular tumor emboli, we speculated that the tumor emboli, which derived from primary lesion, could be associated with the change of adherens junctions in IMPC.

Plakoglobin, a key component in cell adhesion, contributes to both adherens junctions and desmosomes. It has been reported that plakoglobin has oncogenic activity, and is correlated with poor prognosis in patients [17-20]. Furthermore, there is evidence that plakoglobin plays a more important role in tumor cells than in normal epithelial cells [21]. Studies have shown that cadherin/catenin family proteins are associated with cell cluster formation [27-29]. Therefore, we speculated that plakoglobin in IMPC primary lesions may be a key regulator and promote tumor cluster formation. To validate this hypothesis further, we investigated the expression pattern of plakoglobin in IMPC tissues and a simulated cell model (T47D cell clusters) by immunohistochemistry and immunofluorescence. Plakoglobin was found to be overexpressed both in cytoplasm and cell connections of IMPC clusters.
Importantly, when plakoglobin was knocked down by shRNAs, the IMPC clusters were depolymerized and scattered as single cells. Moreover, the increased metastatic propensity of tumor clusters in our mouse model together with the adverse prognosis of IMPC patients with abundant tumor emboli, further support an important role for IMPC cell clusters in cancer metastasis. Although we have also noted that several studies have shown that the decreased plakoglobin in tumors and cell lines is associated with increased metastasis, survival rate, migration, invasion etc. However, in this study, we emphasized that plakoglobin promoting tumor metastasis is based on the unique histomorphology of IMPC. Overall, the heterogeneous expression pattern of adhesion molecules in the primary lesion may lead to the generation of endogenous tumor clusters, facilitating cancer metastasis.

It is known that anoikis can be induced by loss of cell-cell or cell-extracellular matrix interactions, and the vast majority of tumor cells die during this process [30]. However, resistance to anoikis allows a small fraction of malignant cells to survive and initiate tumor metastasis [31]. Whether tumor emboli are more likely to resist anoikis and promote metastatic progression and what the role of plakoglobin is in this process was unclear. Our data in this study showed that the apoptotic ratio was higher in T47D single cells than in T47D cell clusters when cultured in poly-HEMA coated plates and measured by flow cytometry. Consistent with this, T47D single cells in suspension conditions displayed more caspase-3 
A

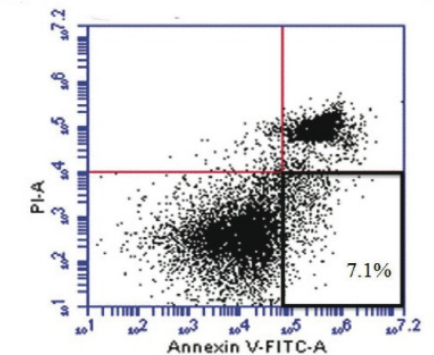

C

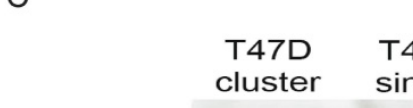

$\mathrm{B}$

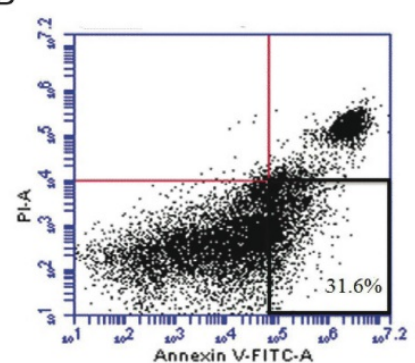

poly-HEMA
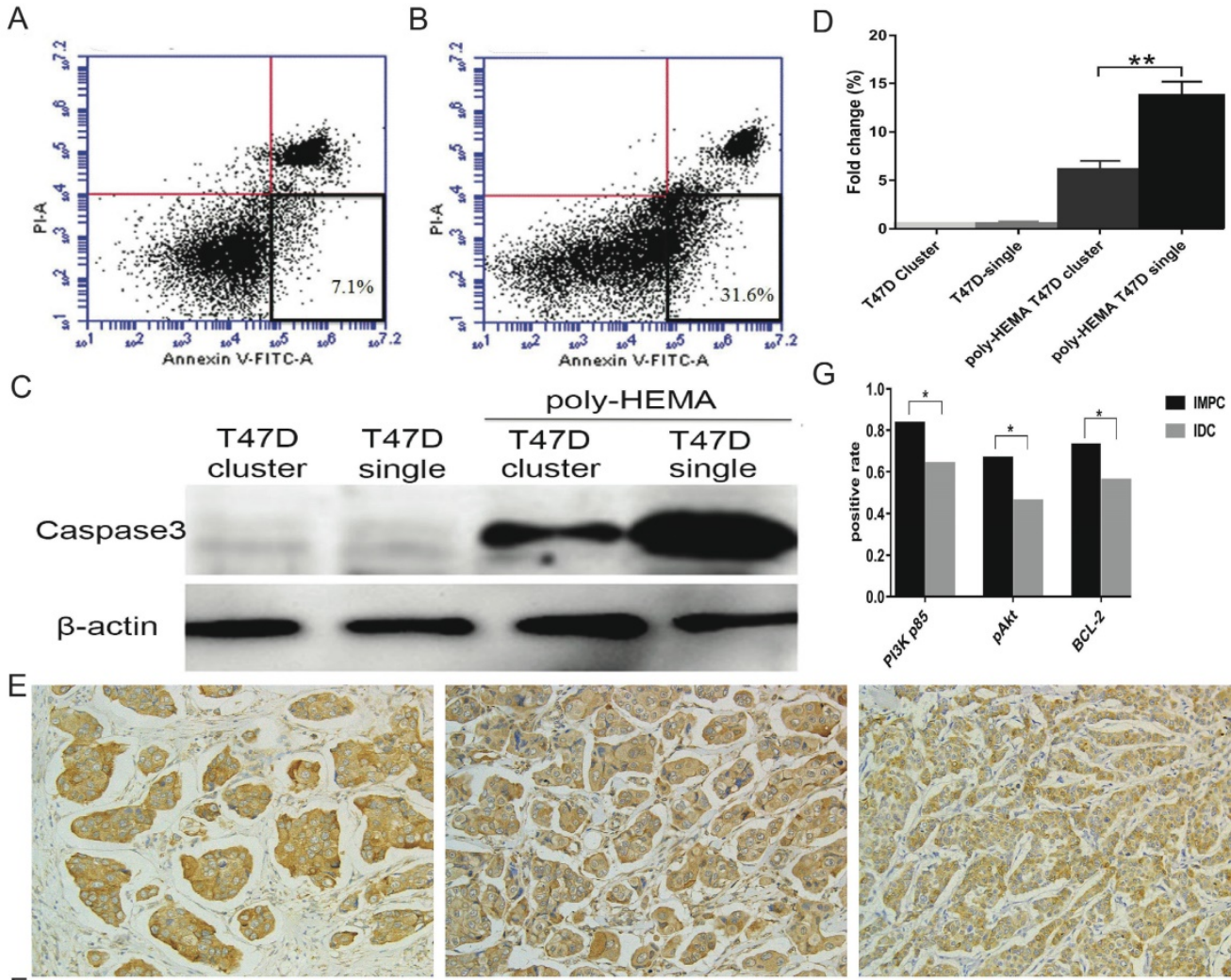

G
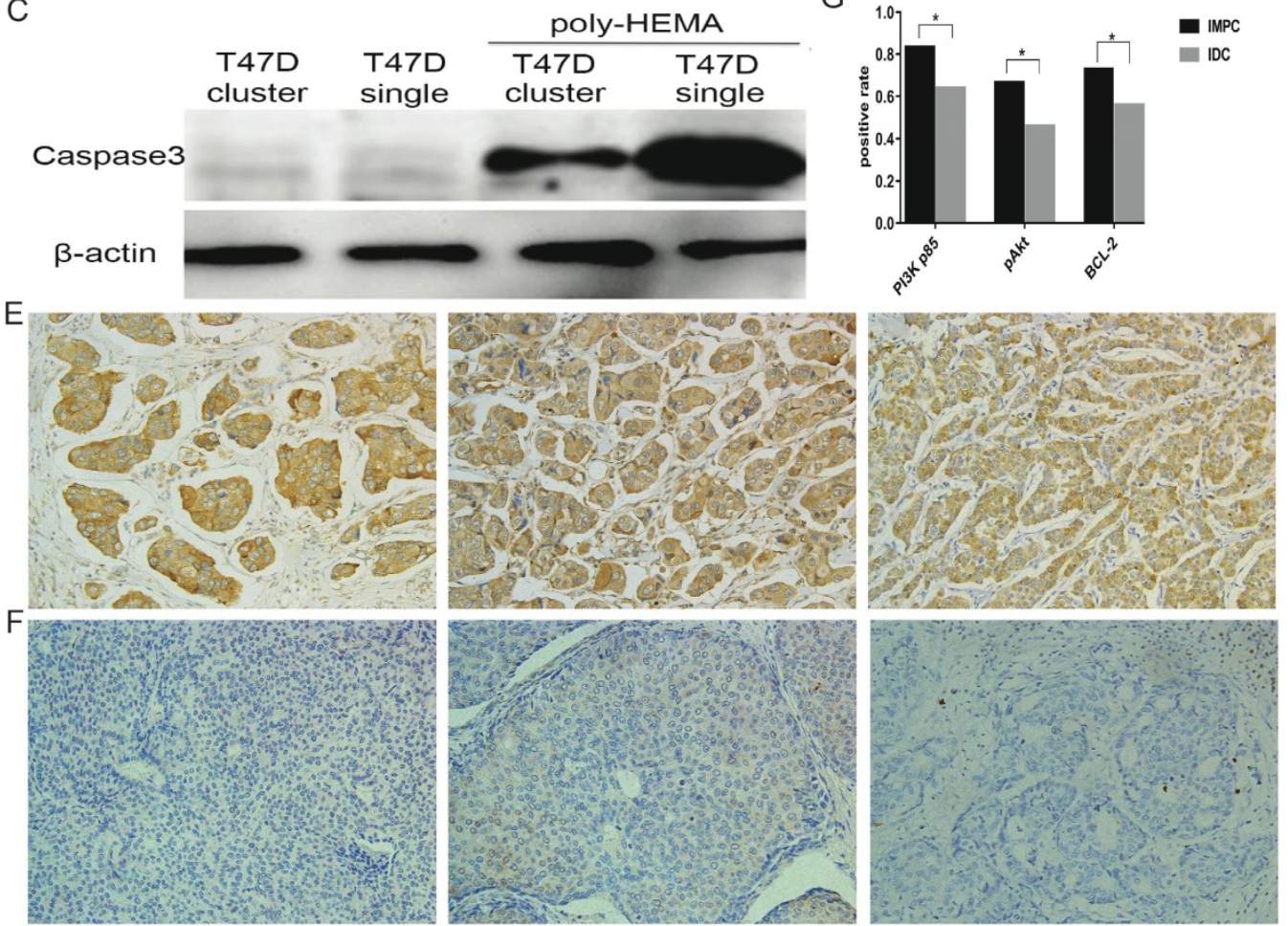

Figure 5. Tumor cells in clusters have a survival advantage under anchorage-independent conditions. A-B: Representative images of apoptotic ratios for T47D cell clusters $(5 \mathrm{~A})$ and single cells $(5 \mathrm{~B})$ under suspension conditions assessed by flow cytometry. Annexin- $\mathrm{V}$ positive and PI negative (at the bottom right in the bivariate correlation plot) are considered as apoptotic cells. C: Immunoblot of cell lysates for T47D single cells or T47D cell clusters analyzed for caspase-3 activation when cultured either in adhesive or suspension plates. D: Error bars represent SEM. $n=3 ;{ }^{* *} \mathrm{p}<0.01$ by student's $t$ test. E: Images of positive expression of PI3K-p85 (left), $\mathrm{PAkt}$ (medium), and Bcl-2 (right) on IMPC cells (200x). F: Images ofnegative expression of PI3K-p85 (left), pAkt (medium), and Bcl-2 (right) on IDC-NST cells (200x). G: Bar graph displaying the positive rate of PI3K-p85, pAkt, and $\mathrm{Bcl}-2$ staining in IMPC and IDC $\left({ }^{*} \mathrm{p}<0.05\right)$.

activity than T47D cell clusters. This further confirms the prolonging survival of tumor clusters in circulation. In other words, tumor cells in clusters are superior to single cells for anoikis resistance. Finally, plakoglobin overexpression was observed in the metastatic LNs of IMPC, suggesting that metastasis is most likely derived from IMPC clusters rather than single cells. Although we cannot exclude the possibility that single tumor cell migration could also contribute to metastasis, our results suggest that high expression of plakoglobin within small clusters of the primary tumor improves the possibility of tumor cells entering the circulation in clusters and retaining their viability.

In addition to regulating cell-cell adhesion, the catenin proteins can also participate in the regulation of cell signaling through interacting with various intracellular molecules. As a member of the Armadillo family of proteins, plakoglobin has well-defined oncogenic and anoikis-resistant potential [32-35]. In Shenhav et.al study, they demonstrated that the plakoglobin binds to the PI3K p85 and promotes PI3K/Akt signaling [36]. Moreover, higher expression of plakoglobin has been shown to induce Bcl-2 expression by promoting nuclear localization of $\beta$-catenin, which activates Myc and EF2 expression. $\mathrm{EF} 2$, in turn, binds to the promoter of and activates Bcl-2 expression [37-39]. It is well documented that plakoglobin can change the level and localization of $\beta$-catenin [40]. Specifically, plakoglobin can replace $\beta$-catenin in the cadherin-catenin complex. The cadherin independent/cytoplasmic $\beta$-catenin can then translocate into the nucleus and regulate the expression of genes involved in proliferation, 
migration, invasion etc. Furthermore, $\beta$-catenin can activate receptor tyrosine kinases, which in turn activate PI3K/Akt pathway that leads to increase signalling $\beta$-catenin levels. Therefore, we presumed that the overexpression of plakoglobin might be involved in the tumor progression. Thus, we evaluated the activity of anoikis-suppression related proteins through western blotting. As expected, results showed that PI3K-p85, pAkt and Bcl-2 were significantly increased in IMPC clusters. By contrast, the plakoglobin level and the activity of the PI3K/Akt/BCL-2 pathway were reduced after transfection with plakoglobin shRNAs. These results indicate that plakoglobin can prevent apoptosis by directly and/or indirectly activating the anti-anoikis pathway. In conclusion, these results provide novel insights into the function of plakoglobin in IMPC metastasis. The high metastatic propensity of IMPC may result from its unique histomorphology of small clusters. The up-regulation of plakoglobin in IMPC might be the culprit that facilitates tumor cluster formation and benefits cluster survival by anoikis-suppression contributing to the process of collective metastasis.

\section{Supplementary Material}

Supplementary figure and tables.

http://www.jcancer.org/v10p2800s1.pdf

\section{Acknowledgements}

\section{Ethics approval and consent to participate}

This study was approved by the Scientific and Ethical Committee of the Cancer Hospital/Institute of Harbin Medical University and all patients provided written informed consent.

\section{Availability of data and materials}

The datasets supporting the conclusions of this article are included within the article and the materials will be available upon request.

\section{Funding}

This study was supported by the National Natural Science Foundation of China (Grant No. 81372839 \& No. 81672599), and the National Science Foundation of Heilongjiang Province of China for Returnees (Grant No. LC2017037).

\section{Authors' contributions}

HL and JHF designed and conceived the study and wrote the manuscript text. HL, JHF and YL conducted the main experiments. NXJ, WYR, LY, XQJ, LLR, ZH, ZXP, LJT, CCW, YY and AWW participated in the experiments and analyzed the data. ZQY supervised the study and provided approval of the manuscript. All authors have read and approved the final manuscript.

\section{Competing Interests}

The authors have declared that no competing interest exists.

\section{References}

[1] Chen L, Fan Y, Lang RG, Guo XJ, Sun YL, Cui LF, Liu FF, Wei J, Zhang XM, Fu L. Breast carcinoma with micropapillary features: clinicopathologic study and long-term follow-up of 100 cases. Int J Surg Pathol. 2008; 16(2):155-163.

[2] Middleton LP, Tressera F, Sobel ME, Bryant BR, Alburquerque A, Grases P, Merino MJ. Infiltrating micropapillary carcinoma of the breast. Modern Pathology. 1999; 12(5):499-504.

[3] Nassar H, Wallis T, Andea A, Dey J, Adsay V, Visscher D. Clinicopathologic analysis of invasive micropapillary differentiation in breast carcinoma. Modern Pathology. 2001; 14(9):836-841.

[4] Pettinato G, Manivel CJ, Panico L, Sparano L, Petrella G. Invasive micropapillary carcinoma of the breast: clinicopathologic study of 62 cases of a poorly recognized variant with highly aggressive behavior. American Journal of Clinical Pathology. 2004; 121(6):857-866.

[5] Wasif N, Maggard MA, Ko CY, Giuliano AE. Invasive lobular vs. ductal breast cancer: a stage-matched comparison of outcomes. Annals of Surgical Oncology. 2010; 17(7):1862-1869.

[6] Chen AC, Paulino AC, Schwartz MR, Rodriguez AA, Bass BL, Chang JC, Teh BS. Prognostic markers for invasive micropapillary carcinoma of the breast: a population-based analysis. Clinical Breast Cancer. 2013; 13(2):133-139.

[7] Chen AC, Paulino AC, Schwartz MR, Rodriguez AA, Bass BL, Chang JC, Teh BS. Population-based comparison of prognostic factors in invasive micropapillary and invasive ductal carcinoma of the breast. British Journal of Cancer. 2014; 111(3):619-622.

[8] Gokce H, Durak MG, Akin MM, Canda T, Balci P, Ellidokuz H, Demirkan B, Gorken IB, Sevinc AI, Kocdor MA, Saydam S, Harmancioglu O. Invasive micropapillary carcinoma of the breast: a clinicopathologic study of 103 cases of an unusual and highly aggressive variant of breast carcinoma. Breast Journal. 2013;19(4):374-381.

[9] Gruel N, Cédenot A, Richardson M, Fréneaux P, Bhalshankar J, Dubois T, Sastre-Garau X, Delattre O, Vincent-Salomon A. Inverted Apical CD24 and Weak EZH2 Expressions Are Phenotypic Characteristics of Pure Invasive Micropapillary Carcinoma of the Breast. Open Journal of Pathology. 2013; 03(02):85-95.

[10] Liu Y, Huang X, Bi R, Yang W, Shao Z. Similar prognoses for invasive micropapillary breast carcinoma and pure invasive ductal carcinoma: a retrospectively matched cohort study in China. PloS One. 2014; 9(9):e106564.

[11] Ma D, Thomas A, Askeland R, Guseva N, Sompallae R. Molecular and immunohistochemical profiling of invasive micropapillary carcinoma of the breast. Pathology and Laboratory Medicine International. 2014:33.

[12] Shi WB, Yang LJ, Hu X, Zhou J, Zhang Q, Shao ZM. Clinico-pathological features and prognosis of invasive micropapillary carcinoma compared to invasive ductal carcinoma: a population-based study from China. PloS One. 2014; 9(6):e101390.

[13] Simonetti S, Terracciano L, Zlobec I, Kilic E, Stasio L, Quarto M, Pettinato G, Insabato L. Immunophenotyping analysis in invasive micropapillary carcinoma of the breast: role of CD24 and CD44 isoforms expression. Breast. 2014; 21(2):165-70.

[14] Yamaguchi R, Tanaka M, Kondo K, Yokoyama T, Kaneko Y, Yamaguchi M, Ogata Y, Nakashima O, Kage M, Yano H. Characteristic morphology of invasive micropapillary carcinoma of the breast: an immunohistochemical analysis. Japanese Journal of Clinical Oncology. 2010; 40(8):781-7.

[15] Acs G,Esposito NN,Rakosy Z,Laronga C,Zhang PJ. Invasive ductal carcinomas of the breast showing partial reversed cell polarity are associated with lymphatic tumor spread and may represent part of a spectrum of invasive micropapillary carcinoma. The American Journal of Surgical Pathology. 2010; 34(11):1637-1646

[16] Siriaunkgul S, Tavassoli FA. Invasive micropapillary carcinoma of the breast. Modern Pathology. 1993; 6(6):660-2.

[17] Chen YJ,Lee LY,Chao YK,Chang JT,Lu YC,Li HF,Chiu CC,Li YC,Li YL,Chiou JF,Cheng AJ. DSG3 facilitates cancer cell growth and invasion through the DSG3-plakoglobin-TCF/LEF-Myc/cyclin D1/MMP signaling pathway. PloS One. 2013; 8(5):e64088

[18] Harris TJ, Tepass U. Adherens junctions: from molecules to morphogenesis. Nature Reviews Molecular Cell Biology. 2010; 11(7):502-14.

[19] Kolligs FT, Kolligs B, Hajra KM, Hu G, Tani M, Cho KR, Fearon ER. gamma-catenin is regulated by the APC tumor suppressor and its oncogenic activity is distinct from that of beta-catenin. Genes \& Development. 2000; 14(11):1319-1331

[20] Pan H, Gao F, Papageorgis P, Abdolmaleky HM, Faller DV, Thiagalingam S. Aberrant activation of gamma-catenin promotes genomic instability and oncogenic effects during tumor progression. Cancer Biol Ther. 2007; 6(10):1638-43. 
[21] Aceto N, Bardia A, Miyamoto DT, Donaldson MC, Wittner BS, Spencer JA, Yu M, Pely A, Engstrom A, Zhu H, Brannigan BW, Kapur R, Stott SL, Shioda T, Ramaswamy S, Ting DT, Lin CP, Toner M, Haber DA, Maheswaran S. Circulating tumor cell clusters are oligoclonal precursors of breast cancer metastasis. Cell. 2014; 158(5):1110-1122

[22] Doublier S, Belisario DC,Polimeni M,Annaratone L,Riganti C,Allia E,Ghigo D,Bosia A,Sapino A. HIF-1 activation induces doxorubicin resistance in MCF7 3-D spheroids via P-glycoprotein expression: a potential model of the chemo-resistance of invasive micropapillary carcinoma of the breast. BMC cancer. 2012; $12: 4$

[23] Yui S,Tomita K,Kudo T,Ando S,Yamazaki M. Induction of multicellular 3-D spheroids of MCF-7 breast carcinoma cells by neutrophil-derived cathepsin G and elastase. Cancer Science. 2005; 96(9):560-570.

[24] Friedl P, Gilmour D. Collective cell migration in morphogenesis, regeneration and cancer. Nature Reviews Molecular Cell Biology. 2009; 10(7):445-457

[25] Bingbing Liu, Xia Zheng, Fanfan Meng, Yunwei Han, Yawen Song, Fangfang Liu, Shuai Li, Lanjing Zhang, Feng Gu, Xinmin Zhang, Li Fu. Overexpression of $\beta 1$ integrin contributes to polarity reversal and a poor prognosis of breast invasive micropapillary carcinoma. Oncotarget. 2018; 9(4):4338-53

[26] Aneta Gandalovicová, Tomás Vomastek, Daniel Rosel, Jan Brábek. Cell polarity signaling in the plasticity of cancer cell invasiveness. Oncotarget 2016; 7(18):25022-49

[27] Alpaugh ML,Tomlinson JS,Kasraeian S,Barsky SH. Cooperative role of E-cadherin and sialyl-Lewis X/A-deficient MUC1 in the passive dissemination of tumor emboli in inflammatory breast carcinoma. Oncogene. 2002; 21(22):3631-3643.

[28] Alpaugh ML, Tomlinson JS, Ye Y, Barsky SH. Relationship of sialyl-Lewis $(\mathrm{x} / \mathrm{a})$ underexpression and E-cadherin overexpression in the lymphovascular embolus of inflammatory breast carcinoma. Am J Pathol. 2002; 161(2):619-628.

[29] Tomlinson JS, Alpaugh ML Barsky SH An intact overexpressed E-cadherin/alpha, beta-catenin axis characterizes the lymphovascular emboli of inflammatory breast carcinoma. Cancer Research. 2001; 61(13):5231-5241

[30] Galante JM, Mortenson MM, Bowles TL, Virudachalam S, Bold RJ. ERK/BCL-2 pathway in the resistance of pancreatic cancer to anoikis. J Surg Res. 2009;152(1):18-25

[31] Liotta LA, Kohn E. Anoikis: cancer and the homeless cell. Nature. 2004;430(7003):973-974

[32] Aktary Z, Pasdar M. Plakoglobin: role in tumorigenesis and metastasis. Int J Cell Biol. 2012:189521

[33] Anastas JN. Functional Crosstalk Between WNT Signaling and Tyrosine Kinase Signaling in Cancer. Semin Oncol. 2015; 42(6):820-831

[34] Jamieson C, Sharma M, Henderson BR. Targeting the beta-catenin nuclear transport pathway in cancer. Semin Cancer Biol. 2014; 27:20-29

[35] Kim W, Kim M, Jho EH. Wnt/ $\beta$-catenin signalling: from plasma membrane to nucleus. Biochem J. 2013; 450(1):9-21

[36] Shenhav Cohen, Donghoon Lee, Bo Zhai, Steven P. Gygi, Alfred L. Goldberg. Trim32 reduces PI3K-Akt-FoxO signaling in muscle atrophy by promoting plakoglobin-PI3K dissociation. J Cell Biol. 2014; 204(5):747-58.

[37] Zackie Aktary, Mahsa Alaee, Manijeh Pasdar. Beyond cell-cell adhesion: Plakoglobin and the regulation of tumorigenesis and metastasis. Oncotarget. 2017; 8(19):32270-91

[38] Li Q, Dashwood WM, Zhong X, Nakagama H, Dashwood RH. Bcl-2 overexpression in PhIP-induced colon tumors: cloning of the rat $\mathrm{Bcl}-2$ promoter and characterization of a pathway involving beta-catenin, c-Myc and E2F1. Oncogene. 2007; 26(42):6194-202.

[39] Li L, Chapman K, Hu X, Wong A, Pasdar M. Modulation of the oncogenic potential of beta-catenin by the subcellular distribution of plakoglobin. Mol Carcinog. 2007; 46(10):824-38

[40] Hakimelahi S, Parker HR, Gilchrist AJ, Barry M, Li Z, Bleackley RC, Pasdar M. Plakoglobin regulates the expression of the anti-apoptotic protein BCL-2. J Biol Chem. 2000; 275(15):10905-11. 